\title{
Fibroblast involvement in cardiac remodeling and repair under ischemic conditions
}

\author{
ZENAIDA CEAUŞU ${ }^{1}$, BOGDAN SOCEA ${ }^{2,3}$, MARIANA COSTACHE ${ }^{4,5}$, DRAGOŞ PREDESCU ${ }^{2,6}$, \\ DRAGOŞ ŞERBAN ${ }^{2,7}$, CĂTĂLIN G. SMARANDACHE ${ }^{2,7}$, IRINA PACU ${ }^{8,9}$, HARADJA HORAŢIU ALEXANDRU ${ }^{9,10}$, \\ ANA MARIA DAVIŢOIU ${ }^{11,12}$, FLORENTINA JACOTĂ-ALEXE ${ }^{9}$, CĂTĂLIN CÎRSTOVEANU ${ }^{11,13}$, \\ MIHAI C.T. DIMITRIU ${ }^{8,9}$, LIANA PLEŞ ${ }^{8,14}$ and MIHAI CEAUŞU ${ }^{4}$ \\ ${ }^{1}$ Department of Pathology, 'Sf. Pantelimon' Emergency Hospital, 021659 Bucharest; ${ }^{2}$ Department of Surgery, \\ 'Carol Davila' University of Medicine and Pharmacy, 020021 Bucharest; ${ }^{3}$ Department of Surgery, \\ 'Sf. Pantelimon' Emergency Hospital, 021659 Bucharest; ${ }^{4}$ Department of Pathology, 'Carol Davila' University of \\ Medicine and Pharmacy, 020021 Bucharest; 5 Department of Pathology, University Emergency Hospital, 050098 Bucharest; \\ ${ }^{6}$ Department of Surgery, 'Sf. Maria' Hospital, 011172 Bucharest; ${ }^{7}$ Department of Surgery, University Emergency Hospital, \\ 050098 Bucharest; ${ }^{8}$ Department of Obstetrics and Gynecology, 'Carol Davila' University of Medicine and Pharmacy, \\ 020021 Bucharest; ${ }^{9}$ Department of Obstetrics and Gynecology, 'Sf. Pantelimon’ Emergency Hospital, 021659 Bucharest; \\ ${ }^{10} \mathrm{PhD}$ Fellowship; ${ }^{11}$ Department of Pediatrics, 'Carol Davila' University of Medicine and Pharmacy, 020021 Bucharest; \\ ${ }^{12}$ Department of Pediatrics, 'Doctor Victor Gomoiu' Emergency Children Clinical Hospital, 022102 Bucharest; \\ ${ }^{13}$ Department of Pediatrics, 'Maria Sklodowska Curie' Emergency Children Clinical Hospital, 041451 Bucharest; \\ ${ }^{14}$ Department of Obstetrics and Gynecology, 'Sf. Ioan' Hospital-Bucur, 040294 Bucharest, Romania
}

Received October 23, 2020; Accepted November 24, 2020

DOI: $10.3892 /$ etm.2021.9700

\begin{abstract}
Cardiac fibroblasts play a main role in the physiological turnover of the extracellular matrix, as well as its pathological remodeling. A study was performed on a batch of 23 cases who died of various cardiac complications secondary to scarring myocardial infarctions. The aim of the study was to assess the fibroblast involvement in cardiac repair under ischemic conditions after myocardial infarction. Tissue myocardial samples from the left ventricle were taken from these cases for microscopy examination, in order to investigate the type and degree of fibrosis as well as the presence of cardiac interstitial fibroblasts. Multiple series of histological sections were also performed and examined, along with immunohistochemical analysis. The fibroblasts were diffusely distributed in the interstitium among the residual cardiomyocytes, showing variable expression of vimentin and smooth muscle actin. During cardiac remodeling, there was a successive interstitial deposition, first of reticulin fibers and then of collagen fibers, leading to interstitial fibrosis and myocardial replacement.
\end{abstract}

Correspondence to: Dr Bogdan Socea, Department of Surgery, 'Carol Davila' University of Medicine and Pharmacy, 37 Dionisie Lupu Street, 020021 Bucharest, Romania

E-mail: bogdan.socea@umfcd.ro

Key words: cardiac fibroblast, cardiac remodeling, extracellular matrix, interstitial fibrosis, hypoxic micro-environment
There was a correlation between vimentin and smooth muscle actin expression and collagen deposition. Fibrosis with cardiac remodeling is based on maintaining proliferation capacity of the fibroblast and its capacity of protein synthesis in the extracellular matrix. Under hypoxic ischemic conditions, followed by myocardial infarction, the fibroblast switches phenotype and transdifferentiate into myofibroblast, contributing to the healing by secreting extracellular matrix proteins and collagen deposition, with subsequent cardiac remodeling and regulation of the micro-environment metabolism.

\section{Introduction}

Heart failure is a complex disorder characterized by interstitial fibrosis, chamber remodeling and reduced ventricular compliance. This is due to an excessive deposition of extracellular matrix proteins by cardiac fibroblasts (1).

The cardiac fibroblast is an interstitial cell, predominantly of embryonic epicardial and endothelial origins, which is located within the myocardial interstitium, epicardial and perivascular regions. Physiologically, it is responsible for homeostasis of the extracellular matrix (2).

Fibroblasts are the main effector cells of cardiac fibrosis. The adult mammalian heart contains abundant fibroblasts that expand following injury and can produce large amounts of extracellular matrix proteins. In the dynamic environment of the ischemic heart, the fibroblasts undergo phenotypic changes, turning into various subtypes with functional diversity, such as: Inflammatory fibroblast which secretes cytokines (IL-1, TNF- $\alpha$ ), matrix-degrading fibroblast which secretes 
matrix-metal proteinases (MMP), phagocytic fibroblast, angiogenic fibroblast which may turn into pericyte, myofibroblast which secretes extra-cellular matrix proteins $(3,4)$.

The function of the fibroblast/myofibroblast is modulated by cytokines, hormones and also by mechanical stretch and changes in oxygen availability. In turn, the myofibroblast responses includes changes in cell proliferation, cell migration, extracellular matrix metabolism and secretion of different bioactive molecules and growth factors (5).

In cardiac ischemia followed by myocardial infarction, the fibroblast plays an active role through communication with the cardiomyocytes by direct cell-cell interactions and autocrine/paracrine-mediated pathways (6). Fibroblasts play central roles in two types of fibrosis: Reparative and reactive. Reparative fibrosis is a replacement fibrosis which accompanies cardiomyocyte death, followed by scar formation. Reactive fibrosis is an interstitial and perivascular fibrosis, which is indirectly associated with cardiomyocyte death (6).

Fibroblasts are the main player in form of mesenchymal cardiac cells, together with stem cells, endothelial cells and vascular smooth muscle cells. Scientists have found various stem cells with therapeutic potential. Stem cells have been found in almost all tissues: In the skin (epidermal stem cells), skeletal muscle (satellite stem cells), small bowel (intestinal stem cells), and liver (oval cells). The mucosa of the small bowel is one of the best examples of a rapidly self-renewing tissue (7). Dedicated stem cells, located at the crypt base represent the origin in the renewing process. They provide, by differentiation, several functional mature cells, by migrating from the base of the crypt toward the top of the villi. All these cells are part of the bigger family of adult mesenchymal and stem cells, also present in the gut and other adult tissues $(8,9)$.

The aim of the present study was to assess the fibroblast involvement in cardiac repair under ischemic conditions after myocardial infarction. The findings indicated that these cells play a role in cardiac remodelling.

\section{Materials and methods}

Case selection for human tissue specimens. A retrospective study was performed on a study batch composed of 23 cases with various ischemic heart diseases. Some of the cases had associated conditions: 4 cases with chronic cardiac failure, 9 cases with coronary atherosclerosis, 1 case with hypertrophic cardiomyopathy with left ventricular hypertrophy.

The cases, 20 males and 3 females (sex ratio $~ 5: 1$ ), with ages ranging from 47 to 90 years (mean $=67.7, \mathrm{SD}=12.3$ ), were selected during an interval of 1 year. They were selected for histopathology, in order to assess cardiac remodeling in hypoxic ischemic micro-environment conditions. All of the patients succumbed following long-term cardiac complications, secondary to scarring myocardial infarctions.

The study was performed according to the World Medical Association Declaration of Helsinki and the tissue specimens were collected according to national legislation, using a protocol approved by the local Bioethics Committee of 'Sf. Pantelimon' Emergency Clinical Hospital (Bucharest, Romania). All the patients provided signed informed consent regarding hospitalization, treatment and the possible future publication of data.
Tissue sampling and stains. Tissue samples from the heart were taken for microscopy investigation. The fragments were harvested from different parts of the anterior, lateral and posterior wall of the left ventricle.

The selected tissue samples were fixed in $10 \%$ neutral-buffered formalin ( $\mathrm{pH}$ 7.0) for 24-48 h and paraffin embedded. Sections were cut at $5 \mu \mathrm{m}$ and stained with standard hematoxylin and eosin (H\&E).

Additional special stains such as Gomori silver stain (for reticulin fibers), elastic van Gieson and Masson's trichrome (for collagen fibers) were carried out. Tissue samples were divided into appropriate-sized slices (3-5 $\mu \mathrm{m})$ for conventional microscopy and immunohistochemistry. Multiple series of histological sections were also performed and examined.

Immunohistochemical analysis (IHC) was performed for vimentin (clone: V9, RTU, Novocastra) and smooth muscle actin (clone: 1a4, RTU, Abcam), using sections displayed on slides treated first with poly-L-lysine. IHC was performed on $3 \mu \mathrm{m}$ sections from formalin-fixed paraffin-embedded specimens. The method used was an indirect tristadial Avidin-Biotin-Complex technique, with a NovoLink Polymer detection system which utilizes a novel control polymerization technology to prepare polymeric HRP-linker antibody conjugates, according to the manufacturer's specifications (Novocastra, UK). Antigen retrieval technique (enzymatic pre-treatment) was carried out, according to the producer's specifications.

All the slides were examined and photographed on an AccuScope Imager microscope (AccuScope ${ }^{\circledR}$ ). Digital images obtained with an incorporated software program were processed and analyzed with Microsoft Office Picture Manager (Washington, DC), running under Windows 10.

Statistical analysis. Statistical analysis was carried out using SPSS version 20 (IBM). Data were analyzed using the Student's $\mathrm{t}$-test, to determine the median, as well as mean \pm standard deviation (SD). $\mathrm{P}<0.05$ was considered statistically significant.

\section{Results}

In the cases from the study batch with previous pathological conditions of myocardial infarction, all tissues had collagen scars, ranging from granulation tissue in various stages of evolution to heavy sclerosis with hyalin deposition, located mainly as intramural or trans-mural. These lesions were accompanied by compensatory hypertrophy of the residual 'stunned' hibernating peri-necrotic cardiomyocytes.

There were also associated lesions in all cases from the study batch, such as variable degrees of interstitial and perivascular fibrosis, ranging from mild collagen deposition to severe sclerosis, with disorganized myocardial fibers (which sustains a variable ischemic cardiac disease), interstitial vasodilation with hyperemia (which sustains various degrees of cardiac failure) and hypoxic myocardial lesions, in form of 'wavy fibers' cardiomyocytes, along with interstitial lipomatosis and focal degeneration of myocardial fibers of granular vacuolar type.

There was an interstitial deposition in the residual myocardium, first of collagen type III (reticulin fibers) which is brittle, then it was replaced in time with collagen type I or II, which is more sturdy. This led to substitution fibrosis and replacement 


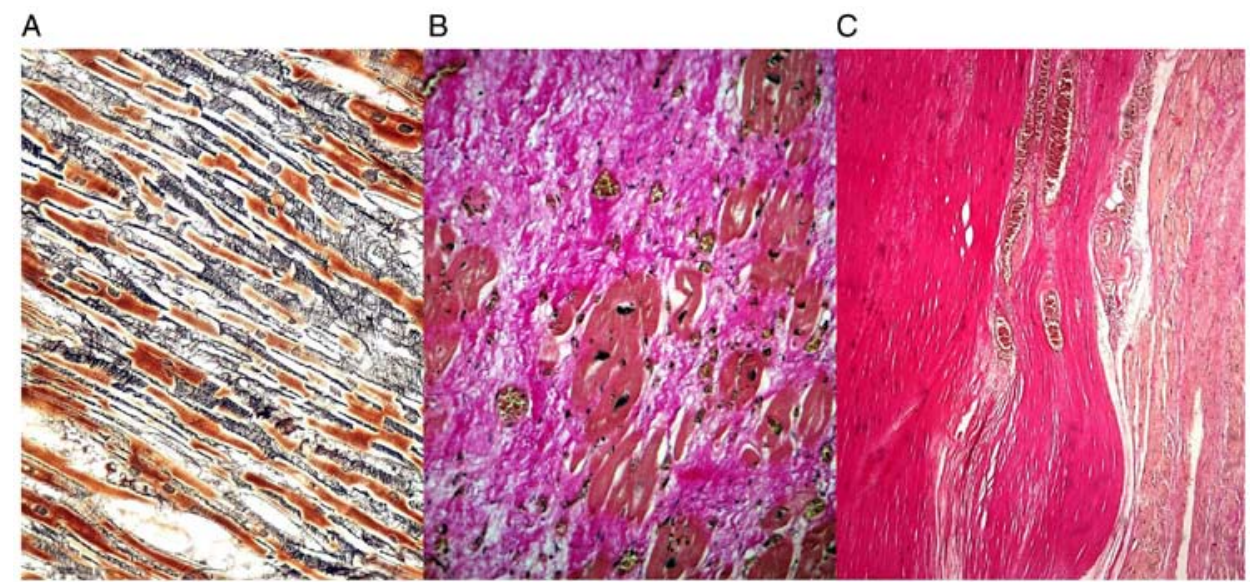

Figure 1. (A) Interstitial fibrosis due to reticulin deposition in a loosely arranged network among the residual cardiomyocytes-Gomori silver stain. (B) Substitution fibrosis and organization, with collagen deposition after a myocardial infarct of about 2-3 weeks-Masson's trichrome. (C) Complete replacement fibrosis with scar formation, with densely, compact parallel collagen fibers in an old myocardial infarct more than 4 weeks-elastic van Gieson.

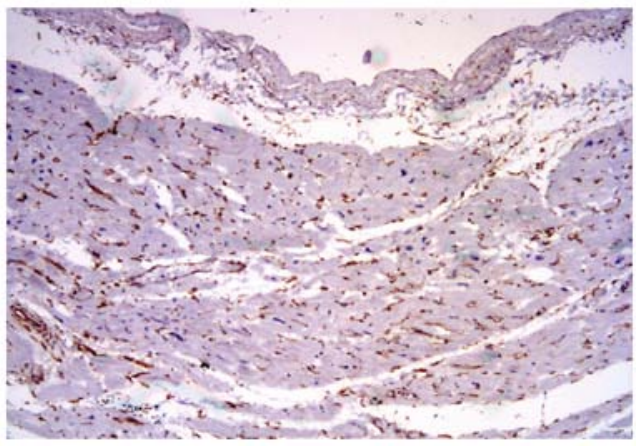

Figure 2. Vimentin expression in the interstitial stroma and vessels in the residual myocardium, staining numerous fibroblasts. IHC, x100. IHC, immunohistochemical analysis.

myocardial fibrosis with scar formation, depending on the circumstances.

The reticulin fibers constituted an interstitial continuous loose network perpendicular on the myocardial cells, while the collagen fibers were more densely, compactly distributed, parallel with the cardiomyocytes (Fig. 1).

The universal marker for fibroblast is vimentin, which showed an unusual expression in the tissue specimens. Vimentin was variably expressed in the interstitial stroma and capillary vessels (Fig. 2), depending on the age of the collagenous scar or the long-term hypoxia.

On the other hand, vimentin was almost completely negative in the fibrous areas or with a mild deposition next to the scar lesions in the extracellular matrix and in the fibroblast/myofibroblast cells.

The smooth muscle actin was also frequently expressed in perivascular myofibroblasts among the residual cardiac fibers and in small vessels (Fig. 3). There was also a direct correlation between vimentin and smooth muscle actin expression and collagen deposition.

\section{Discussion}

In adult humans, the cell populations of the heart are represented by myocytes ( $75 \%$ of the total cardiac volume, but

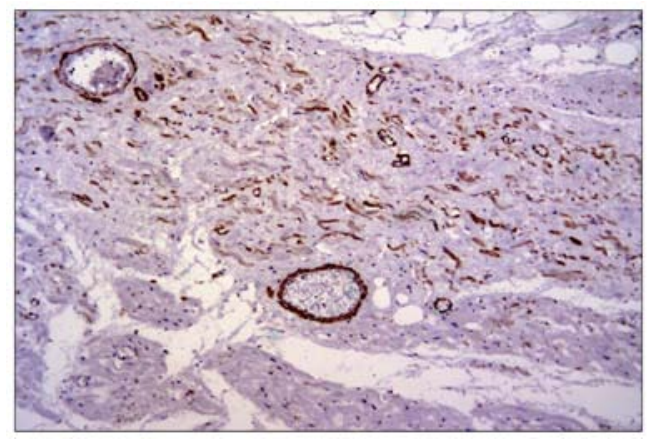

Figure 3. Smooth muscle actin expression in frequent myofibroblasts and vessels among residual ischemic cardiomyocytes after a myocardial infarction. IHC, x100. IHC, immunohistochemical analysis.

only $30 \%$ of the total number of cells), fibroblasts (5\% of the cardiac volume, but $70 \%$ of the total number) and other cells (endothelial cells, smooth muscle cells, vegetative nervous cells and epicardial cells) (10).

Fibroblasts are diffusely located in the normal heart, surrounding the myocytes, playing a role in collagen production and deposition. In addition, they contribute to cardiac development and structural modeling, cell signaling and electric and mechanic functions of the normal and pathological myocardium (11).

Concerning fibroblasts, vimentin is strongly expressed in the fetal heart, but decreases in intensity during life time (accompanied by a constantly increasing expression of desmin). The cardiac fibroblasts are in contact with collagen, other fibroblasts, and myocytes forming a mechanosensitive three-dimensional arrangement (11).

Fibrosis with cardiac remodeling is based on maintaining the proliferation capacity of the fibroblast and its capacity of protein synthesis in the extracellular matrix. Under hypoxic ischemic micro-environment conditions, the resident cardiac fibroblasts become activated and produce collagen fibers with subsequent cardiac remodeling. The cardiac remodeling occurs in the presence of matrix metal proteinases (MMP), particularly MMP-9. Angiogenic proliferation occurs simultaneously with high micro-vascular density and with an 
increased variability of immunohistochemical expression of certain adhesion molecules in the extracellular matrix (such as ICAM-1/CD54 or tenascin-X) (12).

Cardiac repair following myocardial infarction may be divided in three distinct, but overlapping phases: The inflammatory, proliferative, and the maturation phase (13).

In the inflammatory phase, the fibroblasts secrete large amounts of pro-inflammatory mediators and proteases (14); in the proliferative phase they undergo myofibroblast trans-differentiation (expressing SMA) and secrete extracellular matrix proteins (15); and in the maturation phase they deactivate, become quiescent and undergo apoptosis.

Cardiac fibrosis is mainly categorized into four types, based on the location and cause (16).

The first type is interstitial fibrosis, which mainly describes the expansion of endomysium and perimysium, and is caused by progressive deposition of extracellular proteins in the interstitial space, leading to cardiomyocyte death (17).

The second type is replacement fibrosis, which occurs due to necrosis of cardiomyocytes and is associated with systolic ventricular dysfunction, hypertrophic cardiomyopathy and myocarditis (17).

A third category is infiltrative fibrosis, which shows infiltration of inflammatory cells in right ventricles of systemic sclerosis-associated pulmonary arterial hypertension (18).

The fourth type, termed endomyocardial fibrosis, is a primary cause of congestive heart failure in children under 2 years of age with idiopathic etiology (although the literature describes infections, autoimmunity, genetic factors, and nutritional deficiencies) (19).

In the light of the aforementioned allegations, the cardiac fibroblast may be a therapeutic target in myocardial remodeling, with certain anti-fibrotic therapies directed against it, such as: ACE inhibitors and angiotensin receptor antagonists, aldosterone antagonists, endothelin receptor antagonists, statins, anticytokine therapies (TNF $\alpha$, IFN- $\gamma$, and TGF- $\beta$ ), inhibitors of extracellular matrix metabolism involving collagen synthesis, matrix metalloproteinases, and plasmin systems as well as novel antifibrotic/antiinflammatory agents and nuclear receptor ligands $(20,21)$.

Anticoagulant therapy plays a crucial role in preventing ischemic conditions (22-24).

Against all odds and adverse outcomes, cardiac fibroblasts play a main role in the physiological turnover of the extracellular matrix, as well as its pathological remodeling. Although these cells are often associated with cardiac fibrosis, it is important to note that the primary function of fibroblasts is tissue repair-which in cases such as myocardial infarction is in fact beneficial, and its interruption would have undesirable outcomes (25-28).

In summary, under hypoxic ischemic conditions, followed by myocardial infarction, the fibroblast switches phenotype transdifferentiate into myofibroblast, contributing to the healing by secreting extracellular matrix proteins and collagen deposition, with subsequent cardiac remodeling and regulation of the micro-environment metabolism.

\section{Acknowledgements}

Not applicable.

\section{Funding}

No funding was received.

\section{Availability of data and materials}

The datasets used and/or analyzed during the current study are available from the corresponding author on reasonable request.

\section{Authors contributions}

$\mathrm{ZC}, \mathrm{MCo}$ and MCe performed the histological examinations and IHC, and had major contributions in writing the manuscript. BS, DP, DS, CGS, IP, HHA analyzed and interpreted the patient data. MCTD, BS, FJA, CC, LP, AMD searched the literature for similar work and articles and contributed to writing the manuscript. All authors read and approved the final manuscript.

\section{Ethics approval and consent to participate}

The study was conducted according to the World Medical Association Declaration of Helsinki, using a protocol approved by the local Bioethics Committee from 'Sf. Pantelimon' Emergency Clinical Hospital (Bucharest, Romania). All patients had previously signed an informed written consent about hospitalization, treatment and a possible future publication of data.

\section{Patient consent for publication}

Not applicable.

\section{Competing interests}

The authors declare no conflict or competing interests.

\section{References}

1. Travers JG, Kamal FA, Robbins J, Yutzey KE and Blaxall BC: Cardiac fibrosis: The fibroblast awakens. Circ Res 118: 1021-1040, 2016.

2. Krenning G, Zeisberg EM and Kalluri R: The origin of fibroblasts and mechanism of cardiac fibrosis. J Cell Physiol 225: 631-637, 2010.

3. Humeres $\mathrm{C}$ and Frangogiannis NG: Fibroblasts in the infarcted, remodeling and failing heart. JACC Basic Transl Sci 4: 449-467, 2019.

4. Iorga RA, Bacalbasa N, Carsote M, Bratu OG, Stanescu AMA Bungau S, Pantis C and Diaconu CC: Metabolic and cardiovascular benefits of GLP1 agonists, besides the hypoglycemic effect (Review). Exp Ther Med 20: 2396-2400, 2020.

5. Porter KE and Turner NA: Cardiac fibroblasts: At the heart of myocardial remodeling. Pharmacol Ther 123: 255-278, 2009.

6. Takeda $\mathrm{N}$ and Manabe I: Cellular interplay between cardiomyocytes and nonmyocytes in cardiac remodeling. Int J Inflam 2011: 535241, 2011.

7. Tan DW and Barker N: Intestinal stem cells and their defining niche. Curr Top Dev Biol 107: 77-107, 2014.

8. Mehedintu C, Brinduse LA, Bratila E, Monroc M, Lemercier E, Suaud O, Collet-Savoye C and Roman H: Does computed tomography-based virtual colonoscopy improve the accuracy of preoperative assessment based on magnetic resonance imaging in women managed for colorectal endometriosis? J Minim Invasive Gynecol 25: 1009-1017, 2018. 
9. Bratila E, Comandasu D, Coroleuca CA, Cirstoiu M, Bohiltea R, Mehedintu C, Vladareanu S and Berceanu C: Gastrointestinal symptoms in endometriosis correlated with the disease stage, ISI Proceedings, XXXVIth National Congress of Gastroenterology, Hepatology and Digestive Endoscopy, Filodiritto Editore, 67, 2016.

10. Camelliti P, Borg TK and Kohl P: Structural and functional characterization of cardiac fibroblasts. Cardiovasc Res 65: 40-51, 2005.

11. Souders CA, Bowers SL and Baudino TA: Cardiac fibroblast: The renaissance cell. Circ Res 105: 1164-1176, 2009.

12. Sivestre JS, Levy BI and Tedgui A: Mechanisms of angiogenesis and remodelling of the microvasculature. Cardiovasc Res 78 : 201-202, 2008

13. Frangogiannis NG: Regulation of the inflammatory response in cardiac repair. Circ Res 110: 159-73, 2012.

14. Saxena A, Chen W, Su Y, Rai V, Uche OU, Li N and Frangogiannis NG: IL-1induces proinflammatory leukocyte infiltration and regulates fibroblast phenotype in the infarcted myocardium. J Immunol 191: 4838-4848, 2013.

15. Shinde AV, Humeres $C$ and Frangogiannis NG: The role of $\alpha$-smooth muscle actin in fibroblast-mediated matrix contraction and remodeling. Biochim Biophys Acta 1863: 298-309, 2017.

16. Ranjan P, Kumari R and Verma SK: Cardiac fibroblasts and cardiac fibrosis: Precise role of exosomes. Front Cell Dev Biol 7: $318,2019$.

17. Frangogiannis NG: Cardiac fibrosis: Cell biological mechanisms, molecular pathways and therapeutic opportunities. Mol. Aspects Med 65: 70-99, 2019.

18. Overbeek MJ, Mouchaers KT, Niessen HM, Hadi AM Kupreishvili K, Boonstra A, Voskuyl AE, Belien JA, Smit EF, Dijkmans BC, et al: Characteristics of interstitial fibrosis and inflammatory cell infiltration in right ventricles of systemic sclerosis-associated pulmonary arterial hypertension. Int J Rheumatol 2010: 604615, 2010.

19. Duraes AR, de Souza Lima Bitar Y, Roever L and Neto MG: Endomyocardial fibrosis: Past, present, and future. Heart Fail Rev 25: 725-730, 2020.
20. Brown RD, Ambler SK, Mitchell MD and Long CS: The cardiac fibroblast: Therapeutic target in myocardial remodeling and failure. Annu Rev Pharmacol Toxicol 45: 657-687, 2005.

21. Socea B, Socea LI, Diaconu CC, Stanescu AM, Grajdeanu IV, Iancu MA, Pantea Stoian A and Bratu OG: Correlation of oral vitamin $\mathrm{D}$ administration with the severity of psoriasis and the presence of metabolic syndrome. Rev Chim (Bucharest) 69: $1668-1672,2018$

22. Laslo CL, Bacalbasa N, Stanescu AMA, Carsote M, Bungau S, Rus M, Bratu OG and Diaconu CC: New oral anticoagulants possible extension to other indications (Review). Exp Ther Med 20: 2401-2405, 2020.

23. Iorga RA, Bratu OG, Marcu RD, Constantin T, Mischianu DLD, Socea B, Gaman MA and Diaconu CC: Venous thromboembolism in cancer patients: Still looking for answers. Exp Ther Med 18: 5026-5032, 2019.

24. Vladu IM, Radu L, Girgavu SR, Baleanu V, Clenciu D, Ene CG, Socea B, Mazen E, Cristea OM, Mota M and Tenea Cojan TS: An easy way to detect cardiovascular risk. Rev Chim (Bucharest) 69: 4229-4232, 2018

25. Fan D, Takawale A, Lee J and Kassiri Z: Cardiac fibroblasts, fibrosis and extracellular matrix remodeling in heart disease. Fibrogenesis Tissue Repair 5: 15, 2012.

26. Tica OA, Tica O, Antal L, Hatos A, Popescu MI, Pantea Stoian A, Bratu OG, Gaman MA, Pituru SM and Diaconu CC: Modern oral anticoagulant treatment in patients with atrial fibrillation and heart failure: Insights from the clinical practice. Farmacia 66: 972-976, 2018

27. Laslo C, Pantea Stoian A, Socea B, Paduraru D, Bodean O, Socea L, Neagu TP, Stanescu AMA, Marcu D and Diaconu C: New oral anticoagulants and their reversal agents. J Mind Medical Sci 5: 195-201, 2018.

28. Diaconu C: Treatment of diabetes in patients with heart failure. In: The 3rd International conference on interdisciplinary management of diabetes mellitus and its complications - diabetes mellitus in internal medicine, INTERDIAB 2017 Proceedings. Serafinceanu C, Negoita O and Elian V (eds.). Bucharest, pp170-177, 2017. 